\title{
MHV Diagrams and Tree Amplitudes of Gluons
}

Institute for Advanced Study, Princeton, NJ 08540 U.S.A.

E-mail: cachazodias.edu

\section{Freddy Cachazo}

Institute for Advanced Study, Princeton, NJ 08540 U.S.A.

E-mail: cachazodias.edu*

Recently, a new perturbation expansion of gauge theories using MHV amplitudes as vertices was introduced. This alternative to Feynman diagrams is known as MHV diagrams. In this talk I discuss the definition of MHV diagrams for tree-level amplitudes of gluons. This new technique gives a simple and systematic method of computing amplitudes of gluons and makes their twistor space structure manifest.

28th Johns Hopkins Workshop on Current Problems in Particle Theory

June 5-8, 2004

JHU, Baltimore, $M D$

${ }^{*}$ Speaker. 


\section{Introduction}

Scattering amplitudes of gluons in gauge theory can be computed straightforwardly using Feynman diagrams. However, in practice this task becomes very difficult, for the number of Feynman diagrams increases rapidly with the number of external gluons. For example, the number goes from 4 diagrams in a $2 \rightarrow 2$ scattering to more than $10^{7}$ diagrams in a $2 \rightarrow 8$ scattering process. For reviews see [1] and [2].

In this talk I will present an alternative to Feynman diagrams introduced in [3] which uses as vertices maximal helicity violating (or MHV) amplitudes [4][5]. Using a given set of amplitudes as vertices serves as an effective resummation of Feynman diagrams which provides simple answers with a relatively small number of diagrams. The new diagrams are called MHV diagrams.

The discovery of MHV diagrams was motivated by a new string theory on twistor space introduced by Witten in [6]. The theory is based on the topological B model with target twistor space. This model when enriched with D-instantons provides a description of the perturbation theory of $\mathcal{N}=4$ super Yang-Mills ${ }^{1}$. At tree-level, amplitudes of gluons in $\mathcal{N}=4$ super Yang-Mills are the same as amplitudes of gluons in QCD. The reason is that neither fermions not scalars can propagate in the internal lines.

\section{Tree-Level Amplitudes of Gluons}

We will compute amplitudes of n-gluons at tree-level. Each external gluon carries the following information: $\left\{p_{\mu}, \varepsilon_{\mu}, a\right\}$, i.e., momentum, polarization vector, and color index. Amplitudes computed by a straightforward application of Feynman rules are given by very complicated results. The main reason is the large number of invariant but redundant quantities one can built by combining the data for each gluon. There are two main simplifications that lead to more manageable expressions, they are known as the color decomposition and the spinor-helicity formalism. See [2] for a recent review and references.

\subsection{Color-Decomposition}

The first step in simplifying the calculation of gluon amplitudes is to strip out the color structure. This can be achieved as follows,

$$
A_{n}^{\text {tree }}\left(\left\{p_{i}^{\mu}, \varepsilon_{i}^{\mu}, a_{i}\right\}\right)=\sum_{\sigma} \operatorname{Tr}\left(T^{a_{\sigma(1)}} \ldots T^{a_{\sigma(n)}}\right) A^{\text {tree }}\left(\sigma\left(p_{1}^{\mu}, \varepsilon_{1}^{\mu}\right), \ldots, \sigma\left(p_{n}^{\mu}, \varepsilon_{n}^{\mu}\right)\right)
$$

where the sum is over all permutations $\sigma$ of $n$ gluons up to cyclic permutations. The amplitudes on the right hand side are known as partial amplitudes. They are computed using color-ordered Feynman rules.

\subsection{Spinor-Helicity Formalism}

The partial amplitudes $A^{\text {tree }}\left(\sigma\left(p_{1}^{\mu}, \varepsilon_{1}^{\mu}\right), \ldots, \sigma\left(p_{n}^{\mu}, \varepsilon_{n}^{\mu}\right)\right)$ can still be very complicated objects. The reason is that there are many possible Lorentz invariant combinations of momenta and polarization

\footnotetext{
${ }^{1}$ It turns out that at one-loop, twistor string theory amplitudes of gluons contain superconformal gravity in addition to $\mathcal{N}=4$ super Yang-Mills [7].
} 

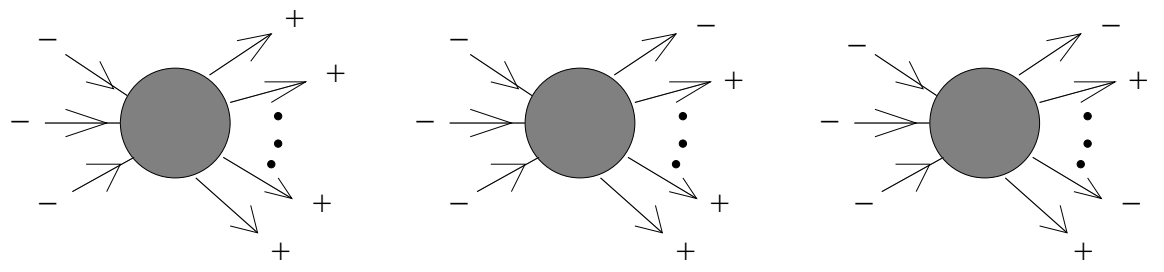

Figure 1: Parke-Taylor amplitudes. From left to right: the first two, $(a)$ and $(b)$, vanish for any number of gluons. The third kind, $(c)$, is non-zero and is known as MHV amplitude.

vectors. The key to simplify this is to realize that in four dimensions, any null vector can be written in terms of two spinors of different chirality. Consider for example the momentum of the $\mathrm{i}^{\text {th }}$ gluon, $p_{i}^{2}=0$, then $p_{a \dot{a}}^{i}=\lambda_{a}^{i} \tilde{\lambda}_{\dot{a}}^{i}$. Likewise polarization vectors can be written in terms of spinors depending on the helicity of the gluon,

$$
\varepsilon_{a \dot{a}}^{(-i)}=\frac{\lambda_{a}^{(i)} \tilde{\mu}_{\dot{a}}}{\left[\tilde{\lambda}^{(i)}, \tilde{\mu}\right]}, \quad \varepsilon_{a \dot{a}}^{(+i)}=\frac{\mu_{a} \tilde{\lambda}_{\dot{a}}^{(i)}}{\left\langle\mu, \lambda^{(i)}\right\rangle}
$$

where $\mu$ and $\tilde{\mu}$ are fixed reference spinors.

Now a given partial amplitude is simply specified by giving $\lambda$ and $\tilde{\lambda}$ for each gluon and its helicity. We denote this by $A_{n}\left(1^{h_{1}}, \ldots, n^{h_{n}}\right)$.

In the end, all amplitudes $A_{n}\left(1^{h_{1}}, \ldots, n^{h_{n}}\right)$ are given as rational functions in the natural inner products of spinors of positive and negative chirality. Take for example the $\mathrm{i}^{\text {th }}$ and the $\mathrm{j}^{\text {th }}$ gluons with $p_{i}=\lambda_{i} \tilde{\lambda}_{i}$ and $p_{j}=\lambda_{j} \tilde{\lambda}_{j}$. The inner products of spinors are defined as follows: $\left\langle\lambda_{i}, \lambda_{j}\right\rangle=$ $\varepsilon_{a b} \lambda_{i}^{a} \lambda_{j}^{b}$ and $\left[\tilde{\lambda}_{i}, \tilde{\lambda}_{j}\right]=\varepsilon_{\dot{a} \dot{b}} \tilde{\lambda}_{i}^{\dot{a}} \tilde{\lambda}_{j}^{\dot{b}}$. In terms of these one can write $2 p_{i} \cdot p_{j}=\langle i, j\rangle[i, j]$.

\subsection{MHV Amplitudes}

The complexity of the amplitudes depends very strongly on the helicity configuration. To see this, consider the process where three negative helicity gluons scatter to produce $n-3$ positive helicity gluons (see figure 1a). Recall that in field theory it is useful to write amplitudes with all legs outcoming, this means that the amplitude in figure 1a is given by $A\left(1^{+}, 2^{+}, 3^{+}, 4^{+}, \ldots, n^{+}\right)$. It turns out that these amplitudes all vanish. The next case is given in figure $1 \mathrm{~b}$, where one of the external gluons has negative helicity, $A\left(1^{-}, 2^{+}, 3^{+}, 4^{+}, \ldots, n^{+}\right)$; these also vanish. Next, consider figure $1 \mathrm{c}$, where two external gluons have negative helicity, say $\mathrm{i}^{\mathrm{th}}$ and $\mathrm{j}^{\text {th }}$ gluons, then

$$
A\left(1^{+}, 2^{+}, \ldots, i^{-}, \ldots, j^{-}, \ldots, n^{+}\right)=i \frac{\langle i j\rangle^{4}}{\langle 12\rangle\langle 23\rangle \ldots\langle n-1 n\rangle\langle n 1\rangle} .
$$

This surprisingly simple formula was conjectured by Parke and Taylor [4] and later proven by Berends and Giele [5]. These are known as maximally helicity violating or MHV amplitudes. The reason for this name should be evident at this point!

\section{MHV Diagrams}

The natural question at this point is whether the simplicity of MHV amplitudes continues to hold as we increase the number of minus-helicity gluons. It turns out that amplitudes with three minuses are complicated and do not reveal any particular structure [8]. 

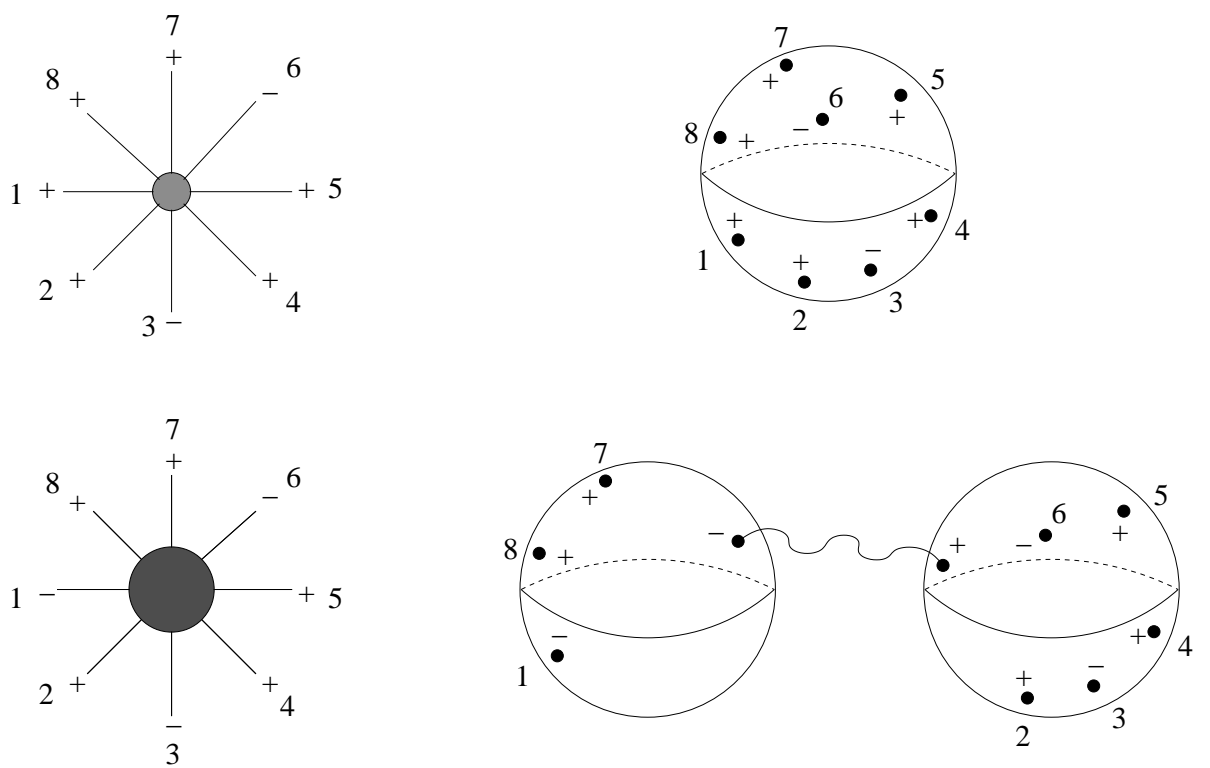

Figure 2: Twistor space structure of tree-level amplitudes of gluons. From top to bottom, (a) an MHV amplitude is localized on a complex line. (b) a next-to-MHV amplitude is localized on the union of two complex lines.

Recently, Witten introduced a twistor string theory whose instanton expansion encodes the perturbative expansion of $\mathcal{N}=4$ super Yang-Mills [6]. Here we are only concerned with tree amplitudes of gluons and supersymmetric partners of the gluons cannot modify the amplitudes.

Twistor space was introduced by Penrose in 1967 [9]. In its simplest version, twistor space is the space with coordinates $\left(Z_{1}, Z_{2}, Z_{3}, Z_{4}\right)=\left(\lambda^{1}, \lambda^{2}, \mu^{1}, \mu^{2}\right)$, with $\mu_{\dot{a}}=-i \partial / \partial \tilde{\lambda}^{\dot{a}}$. It turns out to be natural to mod out by a $\mathbf{C}^{*}$ action which leads to $\mathbf{C} \mathbf{P}^{3}$ instead of $\mathbf{C}^{4}$. Twistor string theory is defined by using the topological B-model which can only admit a Calabi-Yau space as its target space. However, $\mathbf{C P}^{3}$ is not Calabi-Yau. Witten circumvented this problem by adding four Grassman coordinates in order to produce the Calabi-Yau supermanifold $\mathbf{C} \mathbf{P}^{3 \mid 4}$.

One can study how different amplitudes look like when written in twistor coordinates. It turns out that MHV amplitudes vanish unless the twistor coordinates of the gluons lie on a line or $\mathbf{C P}^{1}$. Even more surprising is the fact that amplitudes with three minus helicity gluons, which do not seem to have any particular structure in momentum space, vanish unless the twistor coordinates are on the union of two lines or $\mathbf{C} \mathbf{P}^{1}$ 's. See figure $2 \mathrm{a}$ and $2 \mathrm{~b}$ for examples in eight-gluon amplitudes.

These observations led to the idea that all amplitudes of gluons could be computed by sawing MHV amplitudes, suitably continued off-shell, and connected by some sort of propagator. These are called MHV diagrams [3].

\subsection{Definition of MHV Diagrams}

The precise definition of MHV diagrams is the following: Consider an amplitude with $(q)$ minuses and $(n-q)$ pluses. Draw all possible tree diagrams with $q-1$ nodes and $q-2$ links. Attach external gluons to the nodes (in cyclic order) and helicities to the internal propagators such that each node has the structure of an MHV amplitude with only two minuses. (Everything here 

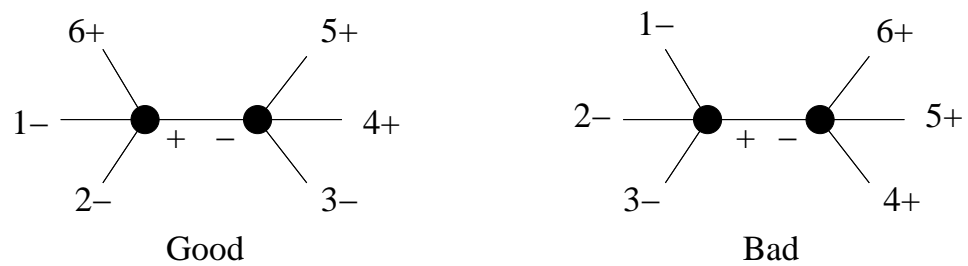

Figure 3: Example of possible external gluon assignments to a tree with two nodes and one link. One the left, one finds MHV like helicities at each vertex, this is an MHV diagram. On the right none of the two vertices has MHV like structure and therefore it should not be considered.

can be repeated using MHV amplitude with only two pluses, the important thing is not to use both at the same time.)

The contribution from each MHV diagrams is computed by the product of MHV amplitudes, one for each node, and scalar Feynman propagators, one for each link.

One immediate problem with this prescription is that the Parke-Taylor formula for MHV amplitudes (2.1) is only valid when the momentum of all gluons is light-like. In a MHV diagram, the momentum $P$ flowing through an internal propagator is not light-like. This means that $P_{a \dot{a}}=\lambda_{a} \tilde{\lambda}_{\dot{a}}+\lambda_{a}^{\prime} \tilde{\lambda}_{\dot{a}}^{\prime}$. In the Parke-Taylor formula we have to give a meaning to $\lambda_{P}$. We choose to define

$$
\lambda_{P a}=P_{a \dot{a}} \eta^{\dot{a}},
$$

where $\eta$ is a fixed negative chirality spinor.

Let us give an example: Consider the six gluon amplitude $A\left(1^{-}, 2^{-}, 3^{-}, 4^{+}, 5^{+}, 6^{+}\right)$. In figure 3 we see two possible diagrams, one of them satisfies all the criteria to be a "good" MHV diagram and the other one fails.

Consider the contribution from the good graph to the amplitude

$$
\frac{\langle 12\rangle^{3}}{\langle 2 P\rangle\langle P 6\rangle\langle 61\rangle} \times \frac{1}{P^{2}} \times \frac{\langle P 3\rangle^{3}}{\langle 34\rangle\langle 45\rangle\langle 5 P\rangle} .
$$

Here $P=p_{6}+p_{1}+p_{2}$ and $\langle P i\rangle$ means $P_{a \dot{a}} \lambda_{i}^{a} \eta^{\dot{a}}$.

\subsection{Proof of The Equivalence to Feynman Diagrams}

Having defined MHV diagrams we now prove that the amplitudes computed from them are well-defined, this means that the amplitudes are independent of the choice of $\eta$.

Once we make a choice of $\eta$, Lorentz invariance is broken. Therefore, proving $\eta$ independence is equivalent to proving that Lorentz invariance is restored. Note that the contribution form each MHV diagram has poles of the form $\langle P, i\rangle$. These poles do not correspond to any physical singularity of the total amplitude and therefore one should prove that they are spurious, i.e., that they have zero residue. Since these poles are at non-Lorentz invariant locations, the proof of Lorentz invariance also implies the vanishing of residues of unphysical poles.

\subsubsection{Lorentz Invariance and Spurious Poles}

The key to prove the $\eta$ independence of the amplitude comes from the twistor string theory construction of the amplitudes. Describing such a construction in detail is beyond the scope of this 
talk. Instead we start by saying that in twistor string theory there is no natural way of defining the null vector $\ell$ associated to $P$. Instead, one has to integrate over all possible choices. Parameterizing $\ell_{a \dot{a}}=\lambda_{a} \tilde{\lambda}_{\dot{a}}$, the integral is given by

$$
\int \frac{\langle\lambda, d \lambda\rangle[\tilde{\lambda}, d \tilde{\lambda}]}{\left(P_{a \dot{a}} \lambda \tilde{\lambda}^{\tilde{a}}\right)^{2}} \times \operatorname{MHV}_{L}(\lambda) \times \operatorname{MHV}_{R}(\lambda)
$$

where $\mathrm{MHV}_{L}$ and $\mathrm{MHV}_{R}$ are the Parke-Taylor amplitudes associated to a given node. Each of them only depends on the positive chirality spinor of external gluons and $\lambda_{P}$ is replaced by $\lambda$. Note that in this integral representation there is no reference to $\eta$, moreover, the result of the integration can only depend on products $\langle i, j\rangle$ and $[i, j]$ of external gluons.

The integral (3.2) has a measure over $\mathbf{C} \mathbf{P}^{1} \times \mathbf{C P}^{1}$ but the contour of integration is over the diagonal $\mathbf{C} \mathbf{P}^{1}$ defined by $\tilde{\lambda}=\bar{\lambda}$. This is the condition for the internal momentum to be real in Minkowski space.

It turns out that this integral is easy to do. One can show that for any function $g(\lambda)$, i.e., independent of $\tilde{\lambda}$,

$$
\frac{[\tilde{\lambda}, d \tilde{\lambda}]}{\left(P_{a \dot{a}} \lambda^{a} \tilde{\lambda}^{\dot{a}}\right)^{2}} g(\lambda)=-d \tilde{\lambda}^{\dot{c}} \frac{\partial}{\partial \tilde{\lambda}^{\dot{c}}}\left(\frac{[\tilde{\lambda}, \eta] g(\lambda)}{\left(P_{a \dot{a}} \lambda^{a} \tilde{\lambda}^{\dot{a}}\right)\left(P_{b \dot{b}} \lambda^{b} \eta^{\dot{b}}\right)}\right) .
$$

Here $\eta$ is any arbitrary but fixed negative chirality spinor. If this identity was true for all $\lambda$ then the integral we want would be zero. However, the equality breaks down where the denominator vanishes in the contour of integration. This happens when $P_{a \dot{a}} \lambda^{a} \eta^{\dot{a}}=0$ and when $\langle\lambda, \bullet\rangle$ in the denominator of $g(\lambda)$ vanishes. The pole $P_{a \dot{a}} \lambda^{a} \tilde{\lambda}^{\dot{a}}=0$ is outside the contour of integration and does not contribute.

The conclusion is that the integral localizes to the location of the poles and it is given by the sum of the residues. The residue at the pole $P_{a \dot{a}} \lambda^{a} \eta^{\dot{a}}=0$ is given by

$$
\operatorname{MHV}_{L}\left(\lambda_{a}=P_{a \dot{a}} \eta^{\dot{a}}\right) \times \frac{1}{P^{2}} \times \operatorname{MHV}_{R}\left(\lambda_{a}=P_{a \dot{a}} \eta^{\dot{a}}\right)
$$

which is exactly the contribution from the MHV diagram prescription.

It turns out that the remaining residues cancel pairwise when the contribution of all integrals is taken into account. This shows that the amplitude computed by adding integrals of the form (3.2), which is $\eta$ independent by definition, is equal to the amplitude computed by adding MHV diagrams. This concludes the proof.

\subsubsection{Collinear and Multiparticle Singularities}

An scattering amplitude has singularities associated to internal particles becoming on-shell. At tree-level, these singularities can only come from propagators that go on-shell. When the momentum in the propagator is given by the sum of two external gluon momenta one says that one has a collinear singularity. When the propagator involves more gluons one has a multiparticle singularity.

Close to any of these singularities, the leading behavior of the amplitude is given by the product of two amplitudes with smaller number of external legs, $A_{L}$ and $A_{R}$, one to the left and one to the right of the internal gluon that becomes on-shell. It is not difficult to show by induction that the amplitudes computed from MHV diagrams reproduce the correct singularities with the corresponding residues. 


\section{Conclusions}

We have introduced an alternative to Feynman diagrams for the computation of tree-level amplitudes of gluons. The new diagrams, called MHV diagrams, are defined by using MHV amplitudes, continued off-shell, as vertices which are connected by Feynman propagators $1 / P^{2}$. Amplitudes computed using MHV diagrams can be shown to be well-defined and to have all the correct singularities of an amplitude computed by Feynman diagrams. This turns out to be enough to conclude that the two are the same since one can complexify the momenta and show that as rational functions of complex variables they are the same. Therefore, we can conclude that MHV diagrams provide a simple and systematic way of computing any tree amplitude of gluons.

\section{References}

[1] M. L. Mangano and S. J. Parke, "Multiparton Amplitudes In Gauge Theories," Phys. Rept. 200, 301 (1991).

[2] L. J. Dixon, “Calculating Scattering Amplitudes Efficiently,” hep-ph/9601359.

[3] F. Cachazo, P. Svrcek and E. Witten, “MHV Vertices and Tree Amplitudes in Gauge Theory," JHEP 0409, 006 (2004) hep-th/0403047.

[4] S. J. Parke and T. R. Taylor, “An Amplitude For N Gluon Scattering,” Phys. Rev. Lett. 56, 2459 (1986).

[5] F. A. Berends and W. T. Giele, Nucl. Phys. B 306, 759 (1988).

[6] E. Witten, "Perturbative Gauge Theory as a String Theory in Twistor Space," Commun. Math. Phys. 252, 189 (2004) hep-th/0312171.

[7] N. Berkovits and E. Witten, "Conformal Supergravity in Twistor-String Theory,” JHEP 0408, 009 (2004) hep-th/0406051.

[8] D. A. Kosower, “Light Cone Recurrence Relations For QCD Amplitudes,” Nucl. Phys. B 335, 23 (1990).

[9] R. Penrose, “Twistor Algebra,” J. Math. Phys. 8, 345 (1967). 